\title{
A Study on the Taboo Search Algorithm for the Open Vehicle Routing Problem with Hard Time Windows
}

\author{
Wang Shuxia \\ Department of computer science \\ Tonghua Normal University \\ Tonghua, china \\ wsx1652@163.com
}

\author{
Xiongying \\ Departmet of computer science \\ Tonhua Normal University \\ Tonghua, china \\ xiongying_fighting@126.com
}

\author{
Wang Zengqiang \\ Qinghe Middle School in Ji'an \\ Ji'an, china \\ Ztong2004@163.com
}

\begin{abstract}
In this paper studying open vehicle routing problem, a taboo search algorithm for the open vehicle routing problem with hard time windows is proposed. After constructing a mathematical model for the problem, this paper focuses on the introduction of the design procedure of TS algorithm for solving the problem. In the searching process of this algorithm, infeasible solution is allowed by adding a penalizing factor, to reduce the probability of local optimal. The computational results are provided. It shows that in most of cases this algorithm can find better solutions.
\end{abstract}

Keywords-open vehicle routing problem, hard time windows, taboo search algorithm

\section{INTRODUCTION}

With the swift development of electric commerce, a large number of enterprises, experts and scholars have focused their study on the improvement and optimization of the logistics distribution system. As an important part of the optimization of logistics distribution system[1,2], VRP

(Vehicle Routing Problem) has become one of the most active and richest themes in the fields of operations science and combination and optimization since it was put forward by some experts like G.Dantzig[3] in1959. OVRP (Open Vehicle Routing Problem)is another type of vehicle routing problem[4], and the main difference from VRP

(Vehicle Routing Problem) is not to ask vehicles to return to the starting station down the original route after taking and sending tasks, or even if remanded to return the original starting station, they still follow the original route to return. Therefore, the running route for vehicles is open, not close. OVRP widely exists in the optimization of logistics distribution and transporting routes of the various transportation means. For example, when it hasn't its own a special fleet or the number of the fleet is not enough, a logistics distribution company will have to rent other vehicles to meet clients' need, because the rented vehicles needn't usually return to the logistics center after finishing tasks.

Time Windows is defined by the fact that clients ask the needed goods to be sent at regulation time. Time Windows can be divided into STW (Soft Time Window) and HTW (Hard Time Window). HTW (Hard Time Window) means that the service for some clients must be carried out at the given time, otherwise they will refuse to accept the service. However, STW (Soft Time Window) means that it is allowable for the company not to provide the service at the given time, but it must compensate the client for the loss caused by fact that it can't provide service at the given time. OVRPHTW (Open Vehicle Routing Problem with Hard Time Windows) is an important expansion of VRP. It adds open constraint conditions to VRP when clients visit the constraints and routes of time windows. The constraint makes the problem description closer to the current situation of logistics distribution, so the study on OVRPHTW is paid more and more attention by people.

\section{THE DESCRIPTION OF OVRPHTW}

OVRPHTW can be described like this: a distribution center provides distribution service for the clients with a certain number ( $\mathrm{n}$ stands for the certain number, $\mathrm{c}=1,2,3 \ldots, \mathrm{n}$ ), and the clients' relevant demanded number is vi $(\mathrm{i} \in \mathrm{C})$. The distribution center owns $\mathrm{k}$ same vehicles $\mathrm{V}=\{1,2, \ldots, \mathrm{k}\}$, load is qi, the number of clients is $\mathrm{N}$, and the location and the demanded number of goods are fixed. Besides, the specific time window is given, and the specific vehicles also own the maximum limit. When they finish the service for the final client, they needn't return to logistics center. The demanded number of clients (vi) is qi $(\mathrm{i}=1,2, \ldots, \mathrm{N})$, and the vehicle running cost between a vehicle park and clients, clients is known. It is made sure that a fleet of running vehicles must meet the following two objects: 1) The number of vehicles serving for all the clients is minimum.2) The whole cost of running vehicles must be minimum. It is necessary to demand an appropriate dispatching scheme of vehicles at time windows. If so, the vehicles in a vehicle 
park will be able to meet all the clients' needs, and the whole cost of vehicles will also be the lowest.

The following is the constraint conditions in the whole process of distribution:

(1) Every route begins with a distribution center, and end up with a client's place.(This is sending goods problem. If it is getting goods problem, it will be vice versa.);

(2) A client can only be served one time and it must be served one time. Moreover, the demanding number should be all met;

(3) The summation of the weight of clients' goods on the distribution route of every distribution vehicle can't go beyond its maximum load.

(4) Suppose a client's time windows is finished by ETi and LTi. If vehicles reach a client ahead of ETi, they need wait; if they get to the place behind LTi, the client $\mathrm{i}$ will refuse to accept the service.

\section{MATHEMATICAL MODELS}

To set up models better, client point number is set as $1,2, \ldots$, N. A decision variable is introduced,

$$
\mathrm{x}_{\mathrm{ij}}{ }^{\mathrm{k}}=\left\{\begin{array}{c}
1, \text { if vehicle } \mathrm{k} \text { from customer (or } \\
0 \text {, else. }
\end{array}\right.
$$

$\mathrm{C}=\{1,2, \ldots, \mathrm{n}\}$ showing clients;

$\mathrm{V}=\{1,2,3, \ldots, \mathrm{k}\}$ indicating a fleet consisting of a group of vehicles with the same type ;

$\mathrm{Cij}$, referring to transportation cost from $\mathrm{i}$ to $\mathrm{j}$, which means distance, cost, and time and so on;

qi( referring to $1,2,3, \ldots \mathrm{N}, \mathrm{i} \neq 0$ ). Every client has their own non-negative demands that it need unload or load goods weight;

$\mathrm{W}$ means the maximum of load weight

In terms of problem description and constraint, a mathematical model based on open vehicle routing problem can be constructed:

$$
\begin{aligned}
& \operatorname{Min} \sum \sum \sum C_{i j} X_{i j}{ }^{k} . \\
& \sum \sum_{X_{i j}{ }^{k}}=1, \forall j \in V \text {; } \\
& k \in C \quad i \in V \\
& \sum_{q_{i}} \sum_{X_{i j}}{ }^{k}<=w, \forall k \in C ; \\
& i \in V \quad j \in V \\
& \sum_{X o_{j k}} \cdot \forall k \in C \text {; } \\
& j \in V \\
& x_{i j}{ }^{k}=0 \text { 或 } 1, \forall i, j \in V, k \in C \text {. }
\end{aligned}
$$

where (1) is the shortest object function of the vehicle route, (2) denotes that each customer can be visited once, (3) is the constraint of vehicle burden, (4) ensure that every vehicle depart from distribution center and not return back to distribution center after each mission,(5) is a strategy variable.

\section{ALGORITHM DESIGN}

According to the description of a model, we can construct the following algorithm:

\section{A. Express solution}

For the model described by OVRPHTW, we can abstractly design a directionless graph. $G, G=(V, E)$, Vertex set $V=\{0,1,2, \ldots, n\}$ denoting the central entrepot and distribution spots, Vertex 0 referring to the central entrepot, Vertex $\mathrm{i}(1 \leqslant \mathrm{i} \leqslant \mathrm{n})$ showing distribution spots. The express for the problem of vehicle distribution can be expressed the permutation of figure $1 \sim \mathrm{n}$ which don't appear repeatedly, including a number of the permutations of 0 . Vertex 0 represents a new distribution route each time it appears.

\section{B. Generated Method of Initial Solution}

Initial solution of taboo search algorithm is the entrance to algorithm. It produces initial solution at random. Since taboo algorithm has a certain dependence on initial solution, the permutation of client service is made at random for many times when initial solution is generated. In a meanwhile, the best permutation of client service is selected to be initial solution.

\section{Method of Generated Neighborhood}

According to algorithm, two exchange methods are adopted to structure neighborhood, that is, two unequal numbers $\mathrm{i}, \mathrm{j}(1 \leqslant \mathrm{i}, \mathrm{j} \leqslant \mathrm{n})$ are produced at random, and then the $\mathrm{i}$ element and the $\mathrm{j}$ element are exchanged in the present solution, thus forming into a solution. The exchange way will produce one of the following two changes at random:

(1) The two elements selected lie in the same route, for instance, $\mathrm{X}=\{01465903287\} \rightarrow$ $\mathrm{X}=\{01564903287\}$

(2) The two elements selected don't lie in the same route, for instance, $X=\{01465903287\} \rightarrow$ $\mathrm{X}=\{01425903687\}$

\section{Taboo List}

Taboo list is used to record the local optimum solution which has been searched for, so that the local optimum solution can emerge in the next searching process, thus searching for the whole space. The design of Taboo list is list structure. Each time a taboo object is added to taboo list, a node is added to the end of the chain list of taboo list. When the length of taboo list is the longest, the oldest node will be deleted from the head of taboo list. Adopting the statics structure of chain list can realize the function of taboo list well. 


\section{E. Termination Condition}

The algorithm uses the prescriptive iteration as termination condition. When the statics is very large, iteration can be added.

\section{F. Value of Objective Function}

In the process of taboo searching, the standard of evaluation on solution must be fixed. In this way, the solution with the better quality can be constantly found in the process of algorithm iteration. In the open vehicle routing problem with hard time windows, the first objective is the least number of vehicles, and the second objective is the least cost of running vehicles.

$$
\sum_{r=1}^{k}[T(r)+p E(r)]
$$

Formation( 6) shows that the constraint condition is put into objective function to measure by introducing a punishment value. $P$ is punishment coefficient .p $\in[0.00001,200000]$ denotes that punishment coefficient equals 1 at the beginning, and it is weighed through a self-adjusting parameter. Iteration is tested every ten times. If the former ten solutions are feasible, it will be divided by 2; If the former ten solutions are not feasible, it will be timed by 2 . This mechanism is put forward when the specialists like Gendreau [5]design the algorithm of the solution to Tavuroute of VRP. It can produce the mixture of a feasible solution and an infeasible one, which is advantageous to reduce the possibility involved in the local optimization. T(r) indicates the cost of runing vehicle, $\mathrm{E}(\mathrm{r})$ indicates the beyond the load weight on the routing $\mathrm{r}$.

\section{EXPERIMENT RESULT AND ANALYSIS}

The authors use the JAVA language program to achieve the above-mentioned taboo search algorithm of open vehicle routing problem with hard time windows, and calculate randomly generated by a computer [initial solution] experimentally.

Example1: suppose the central enrapt of some enterprise need distribute vehicles to its subsidiary twenty-five distribution spots, and rent distribution vehicles from the logistics center belonging to the third side. Meantime, the logistics center belonging to the third side can provide the enterprise with at most twenty-five vehicles of the same type with the largest load of two hundred tons, among which Table 1 below shows the coordinate of the center entrap and distribution spots and the demanded number of the goods.

Using TS algorithm to solve Example1 10 times, the calculated results shown in Table 2. We use the configure of computer is CPU: Celeron D 2.80GHZ, memery: $1 \mathrm{G}$, OS : windows xp sp , compile environment : MyEclipse8.6.

Algorithm Results :What we can get from Table 2: Using TS algorithm for solving Example1 10times, we have got high-quality solutions, the average of the solution is $146.044 \mathrm{~km}$, the average number of vehicles is 4.8 . The fifth and ninth solution has the highest quality, the total distribution distance is $133.61 \mathrm{~km}$, corresponding to the four distribution paths are: path 1: 0-13-17-18-19-15-16-14; path 2 : 0-5-3-7-8-10-11-9-6-4-2; path 3: 0-23-22; path 4: 0-20-24.

\section{SUMMARIES}

The text constructs an improved taboo algorithm to solve OVRPHTW. Through the demonstration of the experiment, the problem of OVRPHTW can be solved effectively, and enhance the practicality of the dispatching system of vehicle distribution.

\section{REFERENCES}

[1] Elliot R, Joseph P B. Physical dist ribution service quality in internet retailing: Service pricing, $t$ ransaction att ributes, and firm att ributes [ J ] . J of Operations Management , 2004, 21 (6) : 6512672.

[2] Niklds A, Fredrik S. Elect ronic commerce, marketing channels and logistics platforms - A wholesaler perspective [ J ] . European J of Operation Research ,2003, 144 (2) : 2702279.

[3] DANTZIG G, RAMSER J . The truck dispatching problem[J ] . Man2agement Science, $1959,(6): 80$ - 91.

[4] Schrage L. Formulation and structure of more complex/realistic routing and scheduling Problems[J] network 1981,11:229-232

[5] Gendreau M, HertzA and LaporteG A tabu search heuristic for the vehicle routing problem[J] Management Sxience,1994,40:1276-1290 
TABLE I EXAMPLE 1 OF THE KNOWN CONDITIONS

\begin{tabular}{lllllll}
$\begin{array}{l}\text { Customer } \\
\text { number }\end{array}$ & $\begin{array}{l}\text { Horizontal } \\
\text { axis(km) }\end{array}$ & $\begin{array}{l}\text { Vertical } \\
\text { axis(km) }\end{array}$ & weight & $\begin{array}{l}\text { Time windows } \\
\text { start time(m) }\end{array}$ & $\begin{array}{l}\text { Time windows end } \\
\text { time (m) }\end{array}$ & $\begin{array}{l}\text { Service } \\
\text { time(m) }\end{array}$ \\
\hline 0 & 40 & 50 & 0 & 0 & 1236 & 0 \\
\hline 1 & 45 & 68 & 10 & 912 & 967 & 90 \\
\hline 2 & 45 & 70 & 30 & 825 & 870 & 90 \\
\hline 3 & 42 & 66 & 10 & 65 & 146 & 90 \\
\hline 4 & 42 & 68 & 10 & 727 & 782 & 90 \\
\hline 5 & 42 & 65 & 10 & 15 & 67 & 90 \\
\hline 6 & 40 & 69 & 20 & 621 & 702 & 90 \\
\hline 7 & 40 & 66 & 20 & 170 & 225 & 90 \\
\hline 8 & 38 & 68 & 20 & 255 & 324 & 90 \\
\hline 9 & 38 & 70 & 10 & 534 & 605 & 90 \\
\hline 10 & 35 & 66 & 10 & 357 & 410 & 90 \\
\hline 11 & 35 & 69 & 10 & 448 & 505 & 90 \\
\hline 12 & 25 & 85 & 20 & 652 & 721 & 90 \\
\hline 13 & 22 & 75 & 30 & 30 & 92 & 90 \\
\hline 14 & 22 & 85 & 10 & 567 & 620 & 90 \\
\hline 15 & 20 & 80 & 40 & 384 & 429 & 90 \\
\hline 16 & 20 & 85 & 40 & 475 & 528 & 90 \\
\hline 17 & 18 & 75 & 20 & 99 & 148 & 90 \\
\hline 18 & 15 & 75 & 20 & 179 & 254 & 90 \\
\hline 19 & 15 & 80 & 10 & 278 & 345 & 90 \\
\hline 20 & 30 & 50 & 10 & 10 & 73 & 90 \\
\hline 21 & 30 & 52 & 20 & 914 & 965 & 90 \\
\hline 22 & 28 & 52 & 20 & 812 & 883 & 777 \\
\hline 23 & 28 & 55 & 10 & 732 & 144 & 90 \\
\hline 24 & 25 & 50 & 10 & 65 & 224 & \\
\hline 25 & 25 & 52 & 40 & 169 & & 90 \\
\hline & & & & & \\
\hline
\end{tabular}

TABLE II EXAMPLE 1 OF THE TS ALGORITHM RESULTS

\begin{tabular}{cccc}
\hline Serial number & Total distance $(\mathbf{k m})$ & Used cars & Running time $(\mathbf{s})$ \\
\hline 1 & 141.81 & 5 & 0.657 \\
\hline 2 & 159.59 & 5 & 0.672 \\
\hline 3 & 150.29 & 5 & 0.687 \\
\hline 4 & 150.62 & 5 & 0.625 \\
\hline 5 & 133.61 & 4 & 0.594 \\
\hline 6 & 151.39 & 4 & 0.672 \\
\hline 7 & 146.74 & 5 & 0.579 \\
\hline 8 & 141.81 & 5 & 0.641 \\
\hline 9 & 133.61 & 4 & 0.631 \\
\hline 10 & 150.97 & 6 & 0.594 \\
\hline average: & 146.044 & 4.8 & 0.6352 \\
\hline
\end{tabular}

